\title{
Comparative Analysis of Platelet-rich Plasma Effect on Tenocytes from Normal Human Rotator Cuff Tendon and Human Rotator Cuff Tendon with Degenerative Tears
}

\author{
Jeong Yong Yoon, Seung Yeon Lee, Sue Shin ${ }^{1}$, Kang Sup Yoon, Chris Hyunchul Jo ${ }^{2 \bowtie}$ \\ Departments of Orthopedic Surgery, ${ }^{1}$ Laboratory Medicine, and ${ }^{2}$ Orthopedic Surgery and Translational Medicine, SMG-SNU Boramae Medical Center, Seoul \\ National University College of Medicine, Seoul, Korea
}

\begin{abstract}
Background: Platelet-rich plasma (PRP) stimulates cell proliferation and enhances matrix gene expression and synthesis. However, there have been no comparative study of the PRP effect on the normal and degenerative tenocytes. The purpose of this study was to compare the effect of PRP on tenocytes from normal and degenerative tendon.

Methods: Tendon tissues were obtained from patients undergoing arthroscopic repair $(n=9)$ and from healthy donors $(n=3)$. Tenocytes were cultured with $10 \%$ (vol/vol) platelet-poor plasma, PRP activated with calcium, and PRP activated with calcium and thrombin. The total cell number was assessed at days 7 and 14 . The expressions of type I and III collagen, decorin, tenascin-C, and scleraxis were evaluated by quantitative real-time reverse transcriptase polymerase chain reaction. The total collagen and glycosaminoglycan (GAG) synthesis was evaluated at days 7 and 14.

Results: No differences were observed between the groups at day 7, but cell proliferation was remarkably increased in tenocytes from the degenerative tendon at day 14. In both tenocyte groups, the gene expressions of type I and III collagen were up-regulated. GAG synthesis was greater in the normal tendon, whereas the expressions of decorin and tenascin-C were increased in tenocytes from the degenerative tendon. Tenocytes from the degenerative tendon had higher fold-change of GAG synthesis and a lower collagen III/I ratio than normal tenocytes.

Conclusions: PRP promoted the cell proliferation and enhanced the synthesis of tendon matrix in both groups. PRP has a greater positive effect on cell proliferation, matrix gene expression and synthesis in tenocytes from degenerative tendon.
\end{abstract}

(Clin Shoulder Elbow 2018;21(1):3-14)

Key Words: Platelet-rich plasma; Tenocytes; Rotator cuff; Tendon

\section{Introduction}

The rotator cuff tendon tear, resulting in impaired shoulder function, are common and account for $30 \%$ to $70 \%$ of all shoulder pain. ${ }^{1,2)}$ Many reports show that the progression of tears is characterized by cellular and extracellular matrix changes including reduced cell number, decreased vascularization, poor matrix organization, changed gene expression and alterations in proteoglycans and collagens; these changes play an important role in rotator cuff tear pathology. ${ }^{3-6)}$ Platelet-rich plasma (PRP) has lately been widely used as a biological solution to improve the tendon-to-bone integration for rotator cuff healing. Currently, a lot of basic sciences and animal data show that PRP positively affects tendon collagen deposition, cell proliferation and matrix

Received November 15, 2017. Revised December 22, 2017. Accepted January 3, 2018.

Correspondence to: Chris Hyunchul Jo

Department of Orthopedic Surgery, SMG-SNU Boramae Medical Center, Seoul National University College of Medicine, 20 Boramae-ro 5-gil, Dongjak-gu, Seoul 07061, Korea

Tel: +82-2-870-2315, Fax: +82-2-870-3864, E-mail: chrisjo@snu.ac.kr

IRB approval: SMG-SNU Boramae Medical Center (No. 26-2015-112).

Financial support: This research was supported by the Basic Science Research Program and the Bio \& Medical Technology Development Program of the National Research Foundation of Korea funded by the Ministry of Science, ICT \& Future Planning (NRF-2015M3A9E6028412 \& NRF-2017R1A2B2010995). Conflict of interests: None. 
synthesis. ${ }^{7-10)}$ Few studies have also reported the effects of PRP on human tendon cells. ${ }^{9,11-13)}$ Recent studies on human tenocyte have investigated the effect of PRP with steroids or triamcinolone acetonide. ${ }^{12,14)}$ In these studies, tenocytes were isolated and cultured from the normal or contractured hamstring tendons, from ruptured achilles tendon, and from human rotator cuff tendon with degenerative tear. ${ }^{9,11-13,15,16)}$ However, there has been no comparative study of PRP effect on tenocytes collected from the same site, between normal and degenerative torn tendon.

The purpose of the study was to compare the effect of PRP on cell proliferation, matrix gene expression and synthesis between normal and degenerated tenocytes. Our hypothesis was that the PRP effect on tenocytes from degenerative tendon is greater than on tenocytes from normal tendon.

\section{Methods}

\section{Isolation and Expansion of Tenocytes from Human Rotator Cuff Tendons}

The study protocol was approved by the Institutional Review Board of SMG-SNU Boramae Medical Center, and tissues were collected after getting informed consent from all subjects. Degenerative tendon specimens were surgically obtained from patients undergoing arthroscopic rotator cuff repair for the treatment of degenerative rotator cuff tears $(n=12)$. Pieces of tissue measuring $3 \times 3 \mathrm{~mm}$ were acquired after debriding the severely frayed portion of the lateral edge of rotator cuff tendons with a basket forceps. Normal tendon specimens were collected during open reduction and internal fixation for the treatment of proximal humerus fracture $(n=3)$. A piece of tissue $(3 \times 3 \mathrm{~mm}$ in size) was obtained from the macroscopically normal rotator cuff tendon after the rotator interval release.

Tenocytes were harvested, isolated and cultured as previously described. ${ }^{7)}$ Totally, 12 patients with rotator cuff tear had shoulder pain with an insidious onset and no history of trauma, whereas 3 patients with proximal humerus fracture had no history of prior shoulder problems. Tendon specimens were washed twice in calcium- and magnesium-free phosphate-buffered saline (Dulbecco's phosphate-buffered saline, DPBS) and finely minced. Cells were released by treating with $0.3 \%$ collagenase II for 2 hours with gentle agitation, in low-glucose Dulbecco's Modified Eagle Medium (LG DMEM) containing antibiotic solution (100 U/ $\mathrm{ml}$ penicillin and $100 \mu \mathrm{g} / \mathrm{ml}$ streptomycin). An equal volume of DPBS was then added, and undigested tissue was removed using a 100-mm nylon sieve. The dissociated cells were collected by centrifugation, washed twice, resuspended in LG DMEM supplemented with $10 \%$ fetal bovine serum (FBS) and antibiotic solution (growth medium), and cultured in 100-mm tissue culture dishes at a density of 2 to $5 \times 10^{4}$ cells $/ \mathrm{cm}^{2}$, at $37^{\circ} \mathrm{C}$ in a humidified $5 \% \mathrm{CO}_{2}$ atmosphere. The medium was replaced twice weekly. When cells reached $60 \%$ to $80 \%$ confluence, they were detached by incubat- ing with $0.25 \%$ trypsin (Welgene, Daegu, Korea) for 10 minutes, washed, and then re-plated at a ratio of 1:3. Cells thus cultured from the second passage to fifth passage were used in the study.

\section{Preparation of Platelet-rich Plasma gel}

PRP $(n=6)$ was obtained from patients undergoing arthroscopic rotator cuff repair using a plateletpheresis system with a leukoreduction set (COBE Spectra LRS Turbo; Caridian BCT, Terumo, Tokyo, Japan), according to the method previously described. ${ }^{17)}$ The target platelet concentration in the final product was $1,400 \times 10^{3}$ platelets/ $\mu$ l. The system was set and primed according to the manufacturer's instructions, using saline solution for priming and anticoagulant acid citrate dextrose solution as the anticoagulant. The complete blood counts were first determined using an aliquot, on a fully automated analyzer (XE-2100; Sysmex Corp., Hyogo, Japan). Platelet counts in PRP were first adjusted with platelet-poor plasma (PPP) to $1,000 \times 10^{3}$ platelets/ $\mu l^{17)}$ and then further diluted or concentrated if necessary. To activate the platelets, 10\% calcium gluconate containing 166.7 IU/ $\mathrm{ml}$ thrombin (Reyon Pharmaceutical, Seoul, Korea) was added to the PPP. Tenocytes were treated with PPP, PRP activated with calcium (PRP-Ca), and PRP activated with calcium and thrombin (PRP-Ca-Thr). Cells were treated with 2\% FBS as controls.

\section{Assay for Tenocyte Proliferation}

Cells were seeded in 24-well plates (SPL Lifesciences, Pocheon, Korea) at a density of $1 \times 10^{3}$ cells $/ \mathrm{cm}^{2} /$ well, and were allowed to attach for 24 hours in LG DMEM supplemented with $2 \% \mathrm{FBS}$ and antibiotic solution. PRP gels (10\% vol/vol) at platelet concentrations of 100, 200,400, 800, 1,000, 2,000, 4,000, 8,000 , or $16,000 \times 10^{3}$ cells $/ \mathrm{ml}$ activated with $10 \%$ calcium gluconate with or without $166.7 \mathrm{IU} / \mathrm{ml}$ bovine thrombin, were then placed on the cell culture insert of each well. Tenocyte proliferation was measured by WST colorimetric assay (EZ-CyTox; Daeil Lab Service, Seoul, Korea) at days 7 and 14, as previously described. ${ }^{7)}$ Each experiment was performed in triplicate.

\section{Assay for Matrix Gene Expression}

Each well of 6-well plates with cell culture inserts (SPL Lifesciences) were seeded with $3 \times 10^{3}$ cells $/ \mathrm{cm}^{2}$, and cells were allowed to attach for 24 hours. PPP or PRP gels (10\% vol/vol) at a platelet concentration of $1,000 \times 10^{3}$ cells $/ \mathrm{ml}$ activated with $10 \%$ calcium gluconate with or without $166.7 \mathrm{IU} / \mathrm{ml}$ bovine thrombin, were placed on the cell culture insert of each well. Real-time reverse transcriptase polymerase chain reaction (RT-PCR) was performed to evaluate the matrix gene expression at days 7 and 14. Total RNA was extracted, and reverse transcription and amplification were performed as previously described, ${ }^{18)}$ using real time RT-PCR (LightCycler 480 Real-Time PCR System; Roche Applied Science, Mannheim, Germany). The following gene specific primer and probe TaqMan gene 
expression assays (Applied Biosystems, Foster City, CA, USA) were then performed: type I collagen (assay ID: Hs00164004_ m1), type III collagen (assay ID: Hs00943809_m1), scleraxis (assay ID: Hs03054634 g1), decorin (assay ID: Hs00266491 $\mathrm{m1}$ ), tenascin-C (assay ID: 1115665 m1), and glyceraldehyde 3-phosphate dehydrogenase (GAPDH) (assay ID: Hs99999905_ $\mathrm{m} 1$ ). All experiments were performed in triplicate, and average values were calculated for normalized expression levels.

During PCR amplification, the amplified product amounts were monitored by continuous measurement of fluorescence. The GAPDH gene used as an internal reference to normalize all RNA expression levels in real time PCR assays (RT-PCR).

\section{Assay for Total Collagen and Glycosaminoglycan Synthesis}

Each well of 6-well plates with cell culture inserts (SPL Lifesciences) were seeded with $3 \times 10^{3}$ cells $/ \mathrm{cm}^{2}$, and cells were allowed to attach for 24 hours. PPP or PRP gels $(10 \% \mathrm{vol} / \mathrm{vol})$ at a platelet concentration of $1,000 \times 10^{3}$ cells/ml activated with $10 \%$ calcium gluconate, with or without $166.7 \mathrm{IU} / \mathrm{ml}$ bovine thrombin were placed on the cell culture insert of each well. The sircol soluble collagen assay (Biocolor, Newtownabbey, UK) was used to quantify the total soluble collagen. Glycosaminoglycan (GAG) was quantified using a method based on 1,9-dimethylmethylene blue assay kit (Biocolor). All assays were performed in triplicate using culture supernatants on days 7 and 14 .

\section{Statistical Analysis}

All values were expressed as mean \pm standard deviation. Independent t-tests were employed to assess differences in PRP effect between the normal and degenerative groups. ANOVA with Bonferroni's multiple comparison post hoc test was used for comparisons of PPP-, PRP-Ca-, and PRP-Ca-Thr-treated cells in each group. All analyses were performed using PASW ver. 18.0 (IBM Co., Armonk, NY, USA). p-values of less than 0.05 were considered statistically significant.
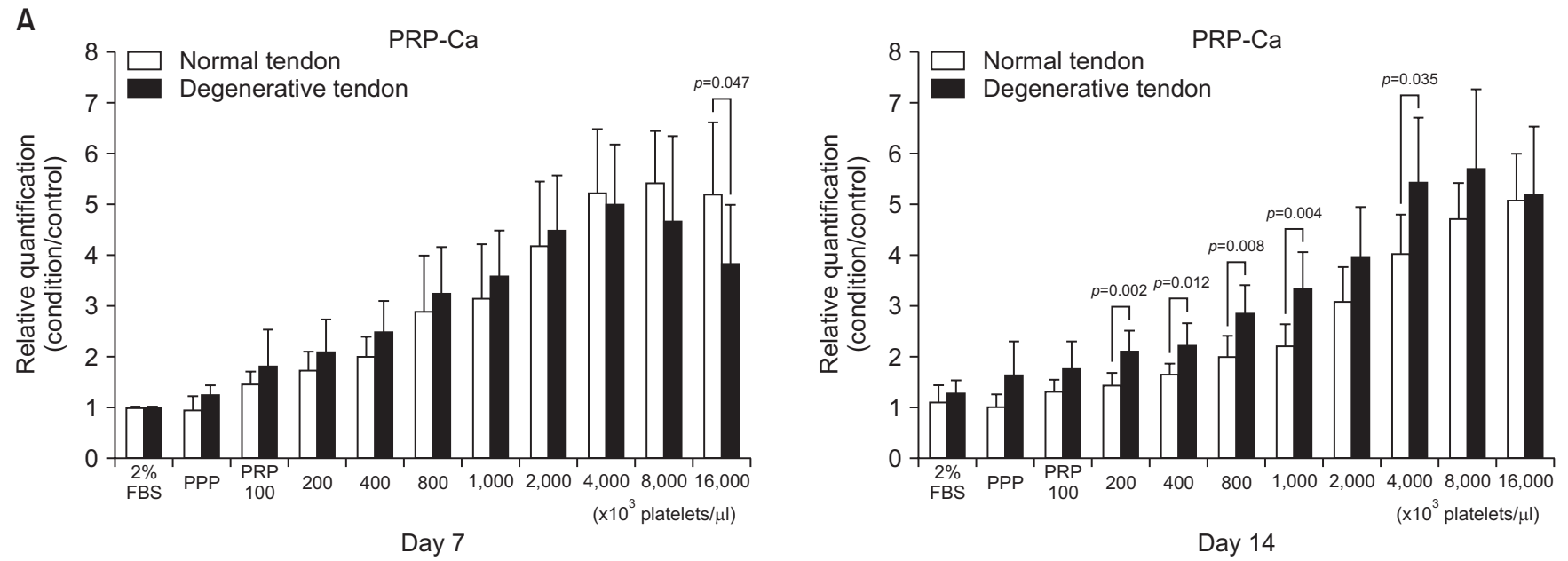

B

PRP-Ca-Thr
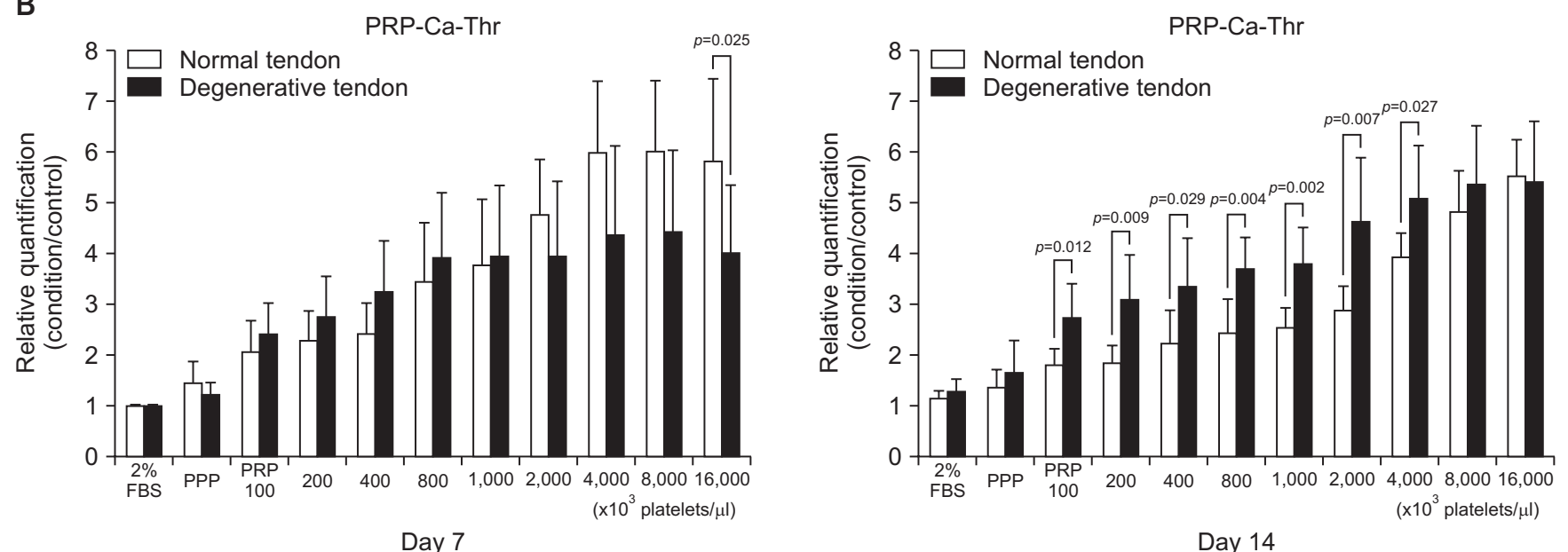

Fig. 1. Relative cell proliferation measured using a WST colorimetric assay (EZ-CyTox assay; Daeil Lab Service, Seoul, Korea). Cells were cultured for 14 days with a platelet-rich plasma (PRP) gel (10\% vol/vol) at platelet concentrations of $100,200,400,800,1,000,2,000,4,000,8,000$, and $16,000 \times 10^{3}$ cells/ $\mu$ l. (A) PRP was activated with calcium only (PRP-Ca). (B) PRP was activated with calcium plus thrombin (PRP-Ca-Thr). 


\section{Results}

\section{Characteristics of Rotator Cuff Tears and Platelet-rich Plasma}

The average age of the 12 patients with degenerative rotator cuff tendon was $58.6 \pm 10.5$ years (range, 39-79 years), and the
3 patients from whom normal rotator cuff tendon was harvested was $71.7 \pm 15.5$ years (range, $54-83$ years). There were no significant differences in age between the groups. The average age of the 6 patients from whom PRP was prepared was $46.3 \pm$ 17.6 years (range, 23-68 years). Platelet counts increased from $202.4 \pm 23.12\left(\times 10^{3}\right.$ platelets/ $\mu$ l) in whole blood to $966.8 \pm$

A
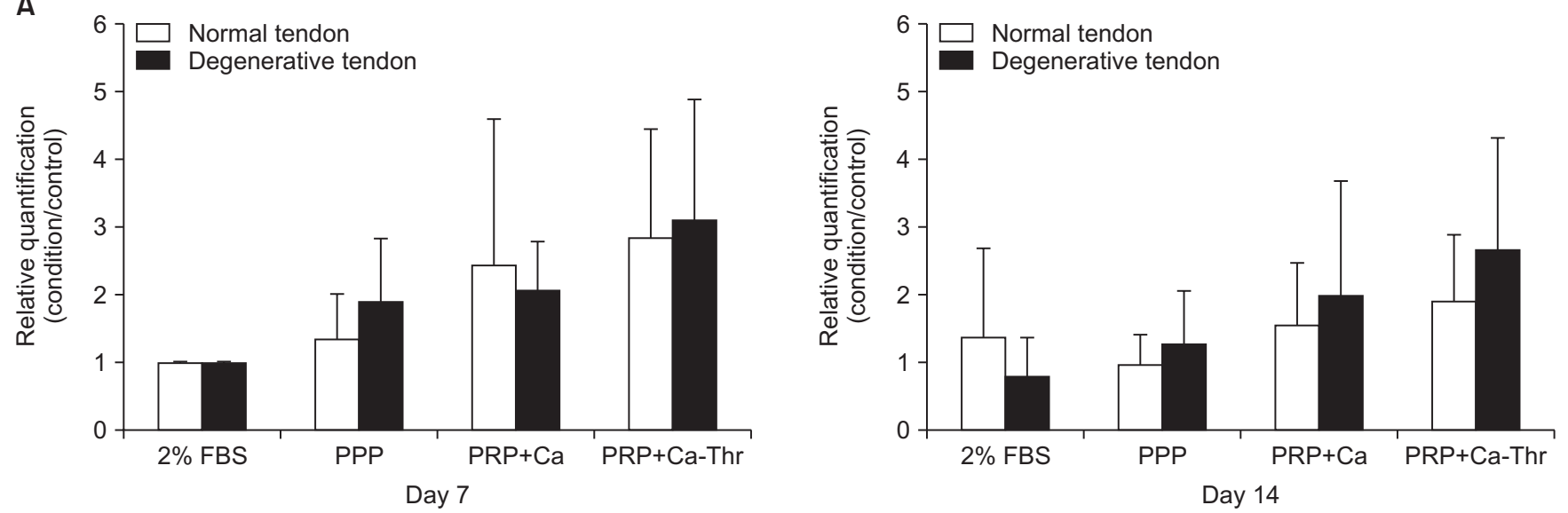

B
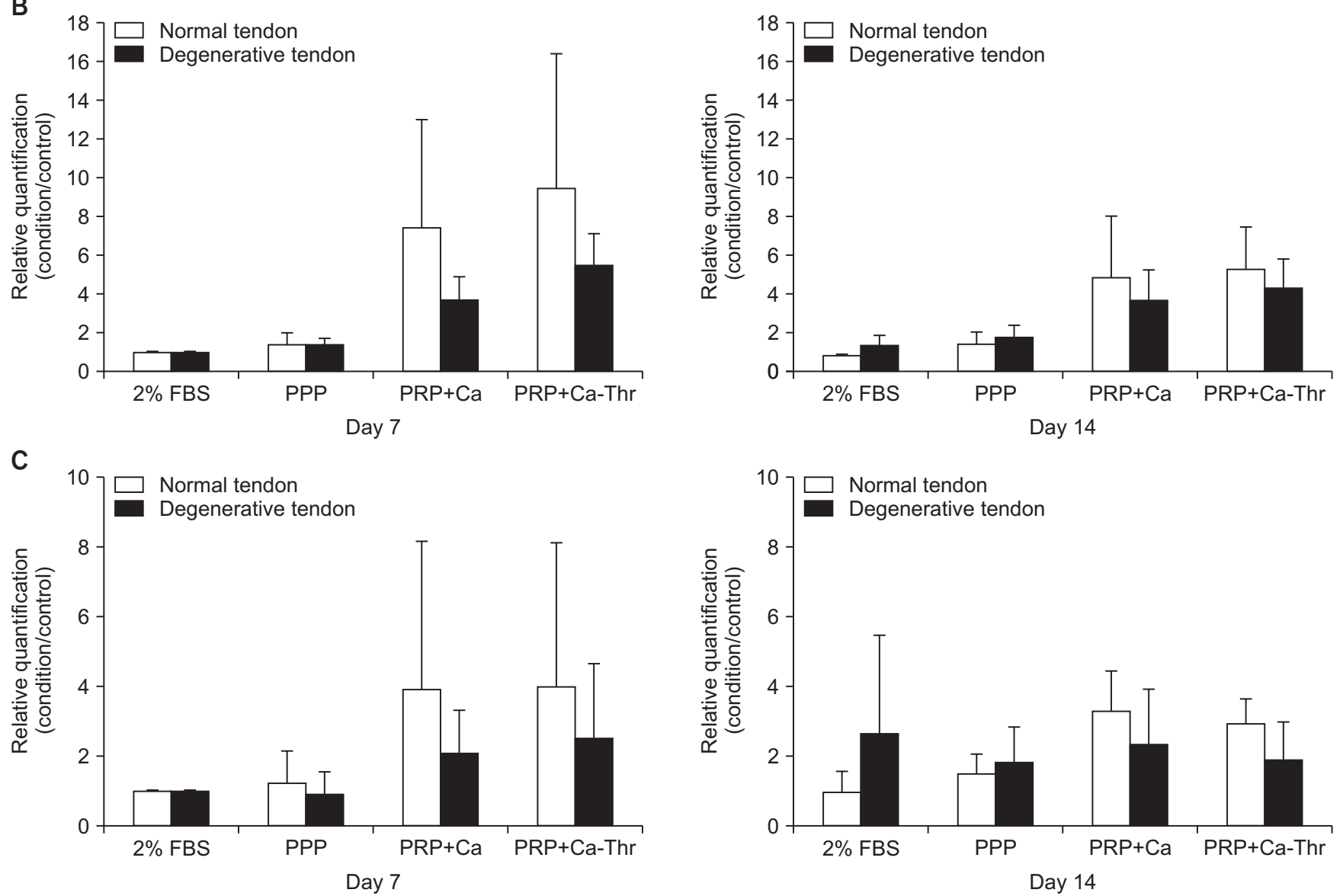

Fig. 2. Relationships between normal group and degenerative group in gene expression levels of type I and III collagen. (A) Gene expression level of type I collagen. (B) Gene expression level of type III collagen. (C) The ratio of the gene expression of type III to I collagen.

FBS: fetal bovine serum, PPP: platelet-poor plasma, PRP-Ca: platelet-poor plasma activated with calcium, PRP-Ca-Thr: platelet-poor plasma activated with calcium and thrombin. 
$56.4\left(\times 10^{3}\right.$ platelets/ $\mu$ l) in PRP, a 4.8-fold increase from baseline $(p<0.001)$. The mean red blood cell and white blood cell counts reduced from $4.62 \pm 0.49$ and $6.25 \pm 1.28$ in whole blood to $0.16 \pm 0.06$ and $0.03 \pm 0.03$ in PRP, respectively $(p<0.001)$. The average concentration of fibrinogen in PRP was $195.72 \pm$ $9.68 \mathrm{mg} / \mathrm{dl}$.

A

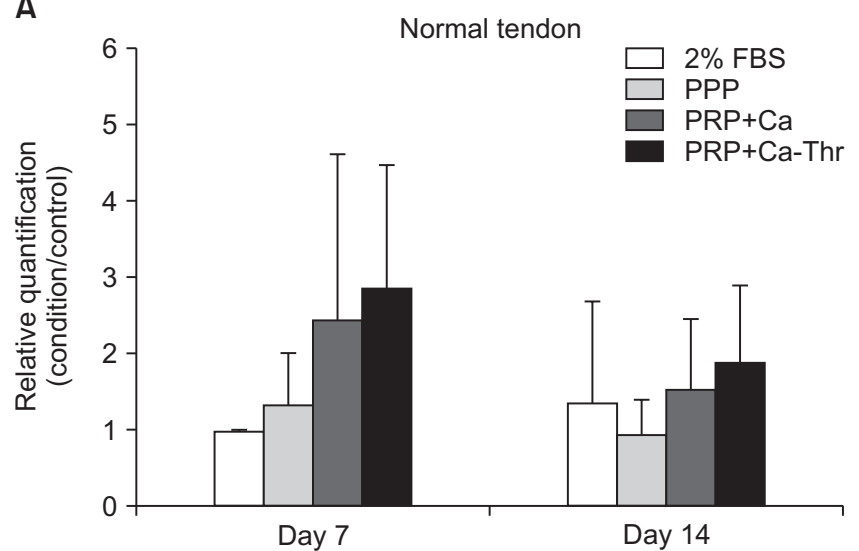

B

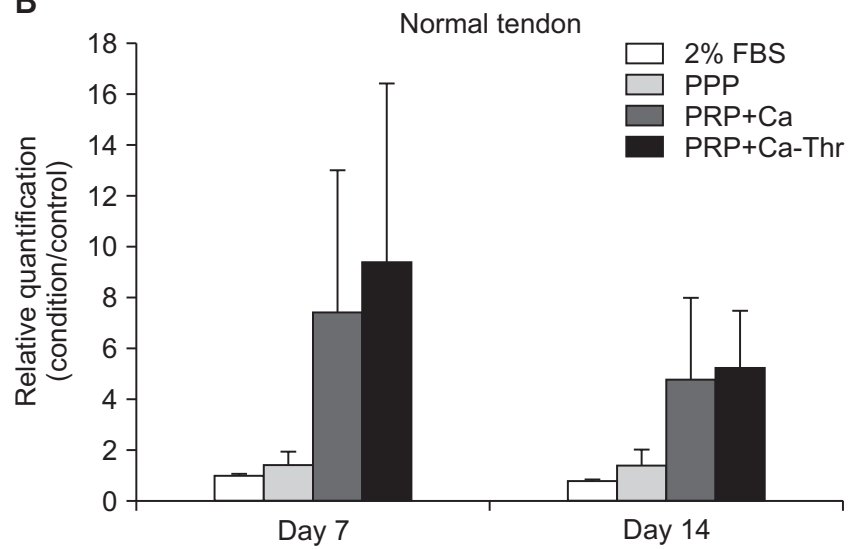

C

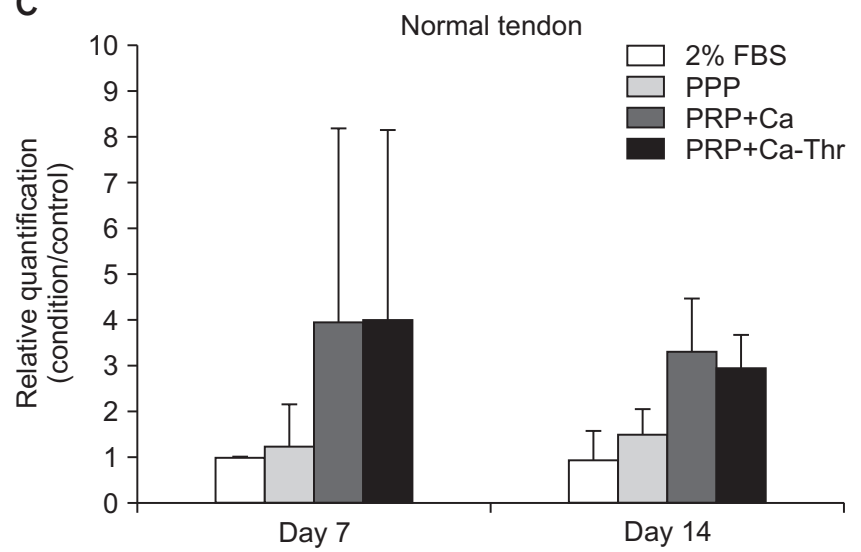

Effect of Platelet-rich Plasma on the Proliferation of Tenocytes between Normal and Degenerated Torn Tendon

At day 7, PRP-Ca with a platelet concentration of $16,000 \times 10^{3}$ cells/ $\mu$ l showed significant difference between normal and degenerative tendon $(p=0.047)$. Activation of PRP

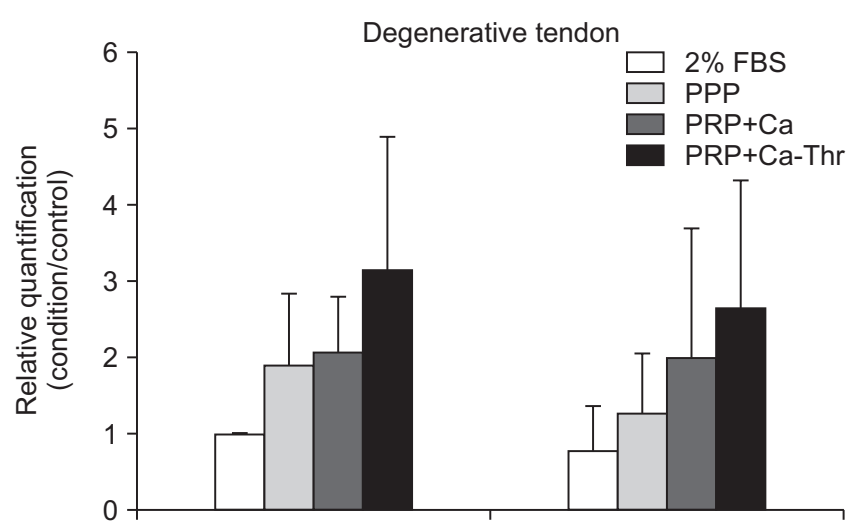

Day 7

Day 14
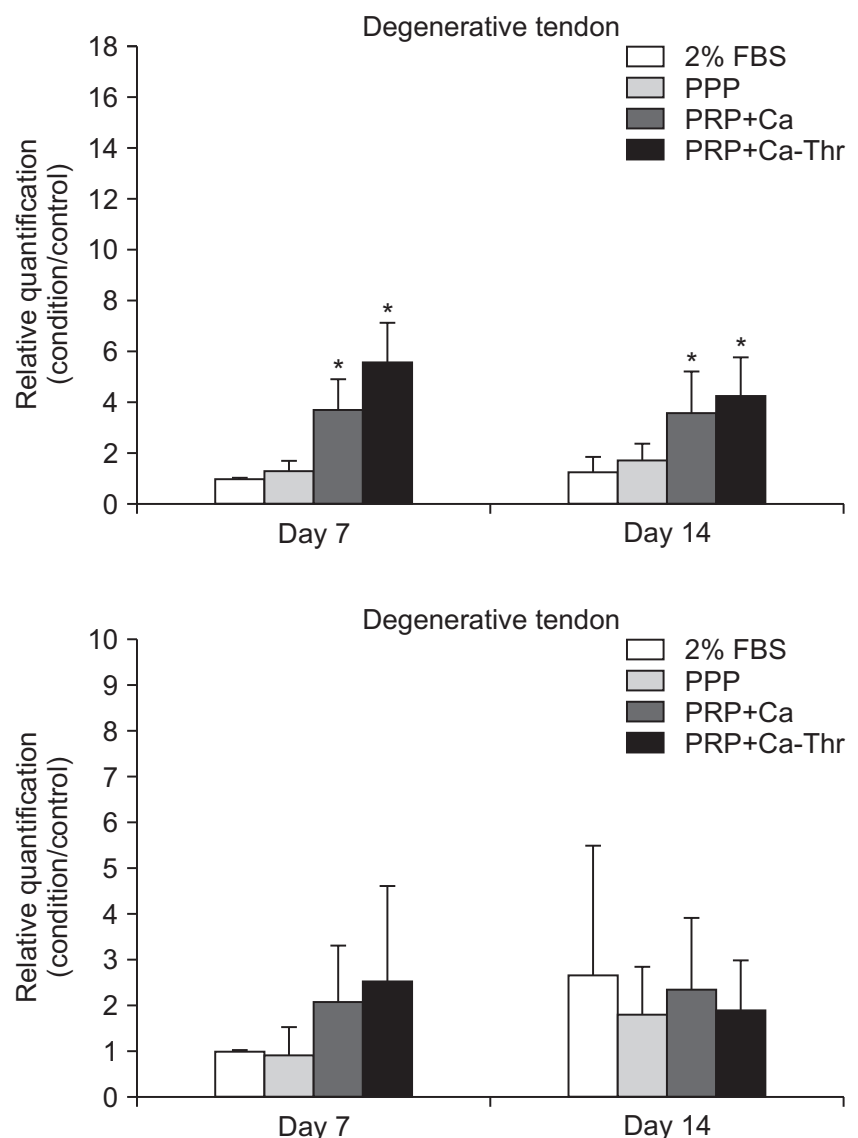

Fig. 3. Gene expression levels of type I and III collagen measured by real-time reverse transcriptase polymerase chain reaction. Relative quantifications were calculated by dividing the mRNA expression level in cells treated with platelet-rich plasma (PRP) by that in control cells. (A) Type I collagen. (B) Type III collagen. (C) The ratio of the gene expression of type III to I collagen.

FBS: fetal bovine serum, PPP: platelet-poor plasma, PRP-Ca: PRP activated with calcium, PRP-Ca-Thr: PRP activated with calcium and thrombin. ${ }^{*}$ Statistical significance at the level of $p<0.001$. 
with calcium promoted cell proliferation in normal tendon at higher concentrations of $4,000,8,000$, and $16,000 \times 10^{3} / \mu$ l. At day 14 , PRP-Ca with a platelet concentration of 200, 400, 800, 1,000 , and $4,000 \times 10^{3} / \mu \mathrm{l}$ showed significant difference between normal tendon and degenerative tendon $(p=0.002, p=0.012$, $p=0.008, p=0.004, p=0.035$, respectively). At these concentrations, the cell proliferation in the degenerative tendon had greater proliferation compared with normal tendon (Fig. 1A). At day 7 , PRP-Ca-Thr at a platelet concentration of $16,000 \times 10^{3}$ / $\mu l$ showed significant difference between normal and degenerative tendon $(p=0.025)$. At day 14 , the PRP-Ca-Thr with a platelet concentration of 100, 200, 400, 800, 1,000, 2,000, and $4,000 \times 10^{3} / \mu$ l showed significant differences between normal and degenerative tendon $(p=0.012, p=0.009, p=0.029$,

A
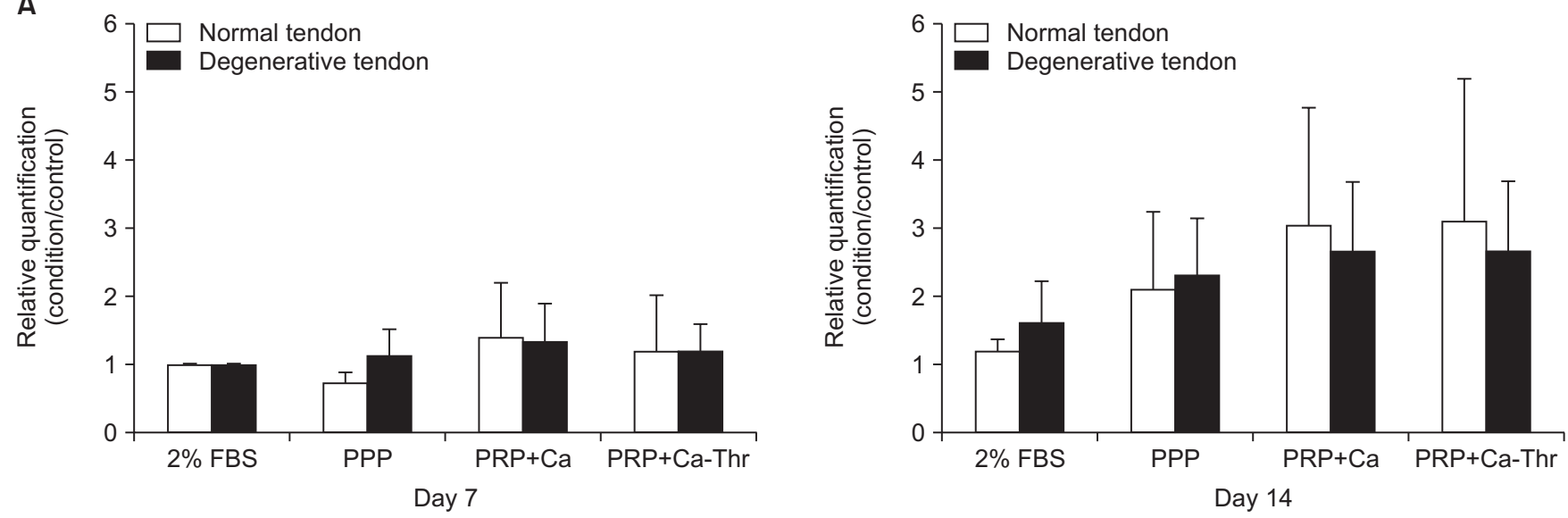

B
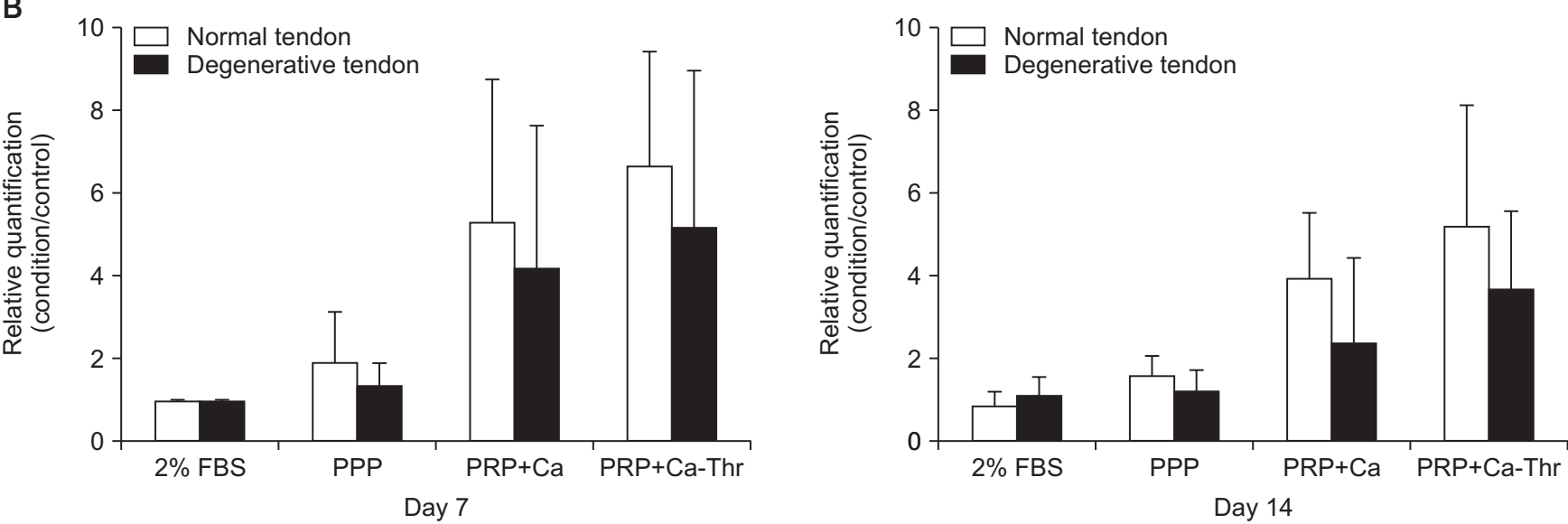

C
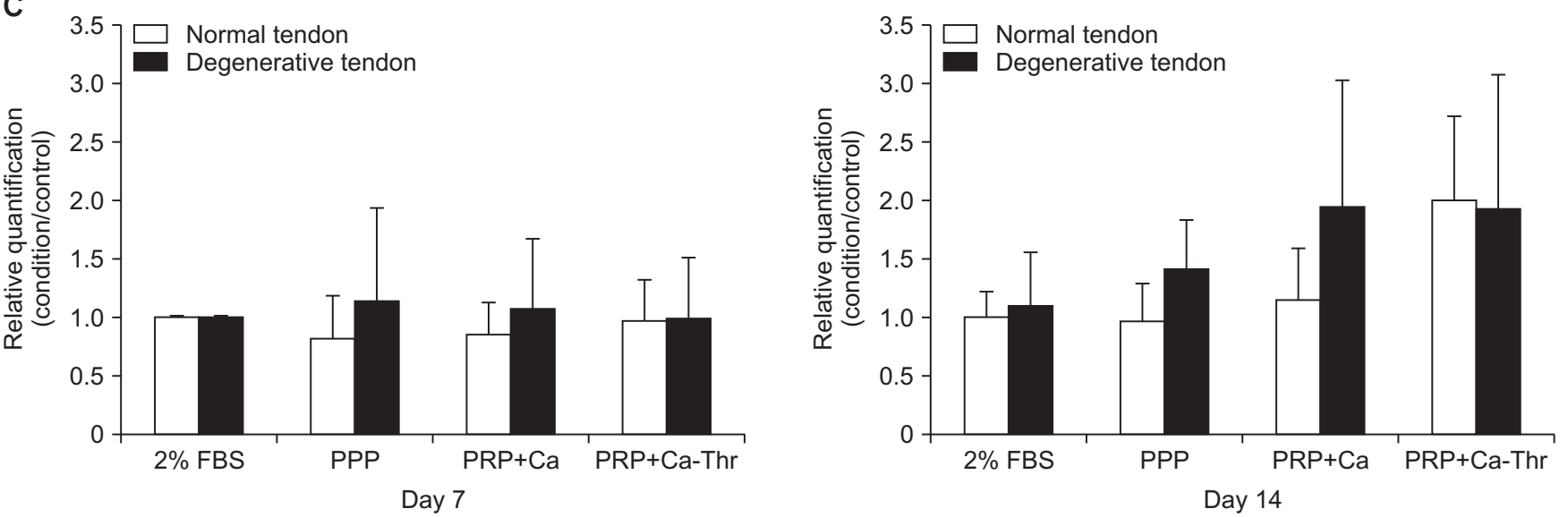

Fig. 4. Relationship between normal group and degenerative group in gene expression levels of decorin (A), tenascin-C (B), scleraxis (C).

FBS: fetal bovine serum, PPP: platelet-poor plasma, PRP-Ca: platelet-rich plasma activated with calcium, PRP-Ca-Thr: platelet-rich plasma activated with calcium and thrombin. 
$p=0.004, p=0.002, p=0.007, p=0.027$, respectively). At these concentrations, the cell proliferation in degenerative tendon was greater as compared to normal tendon (Fig. 1B).

\section{Effect of Platelet-rich Plasma on Matrix Gene Expression of Tenocytes between Normal and Degenerated Torn Tendon}

No significant differences were observed in type I and III col- lagen between the groups (Fig. 2A, B). At day 14, PRP-Ca and PRP-Ca-Thr increased the gene expression of type I collagen in both groups as compared to the control; PRP-Ca and PRPCa-Thr, respectively, upregulated the gene expression by 2.54 and 3.39-fold in degenerated torn tendon, and 1.13- and 1.38fold in the normal tendon (Fig. 3A). The gene expression of type III collagen was significantly induced by PRP activated with calcium and calcium-thrombin in the degenerated torn tendon
A

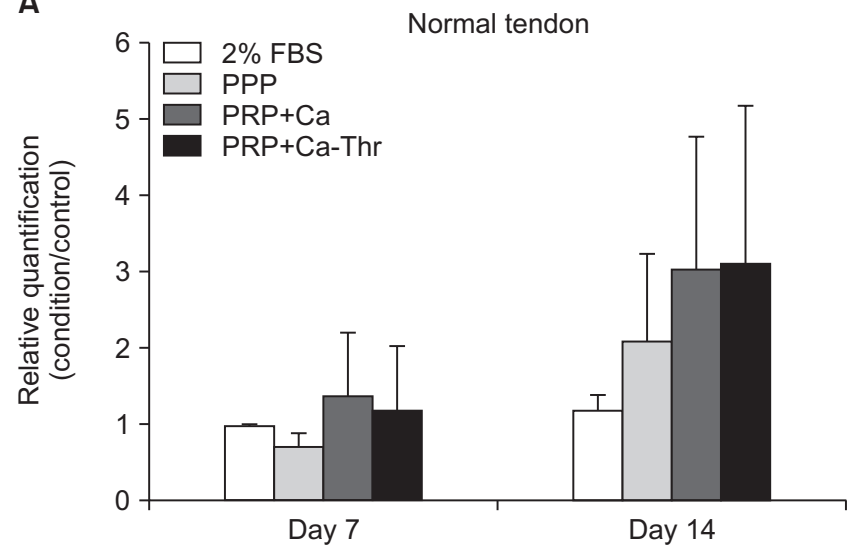

B

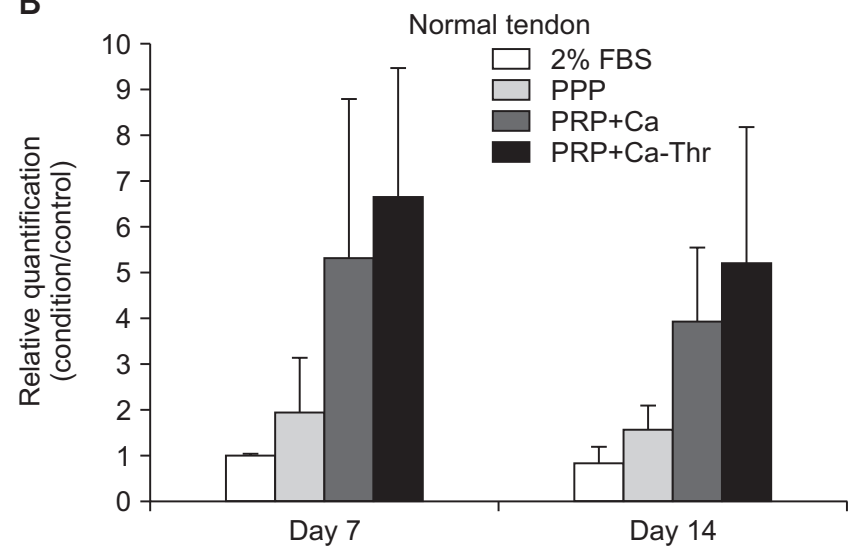

C

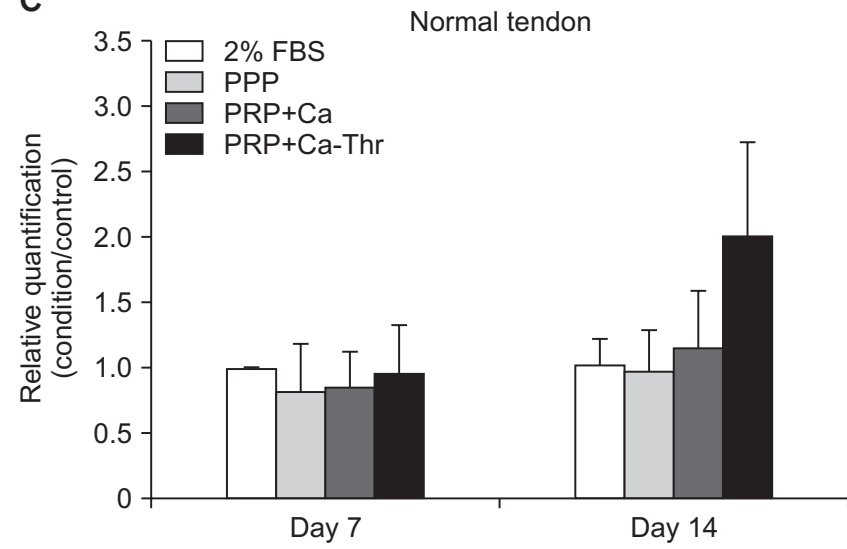

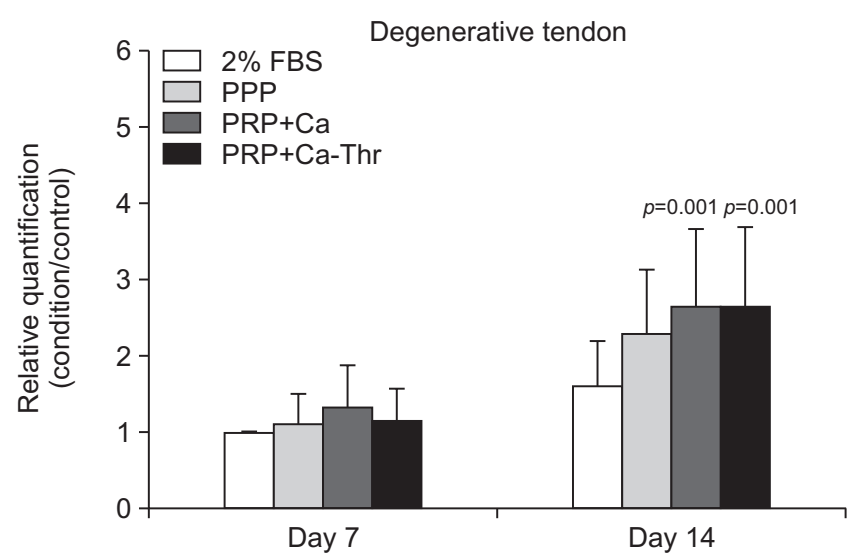
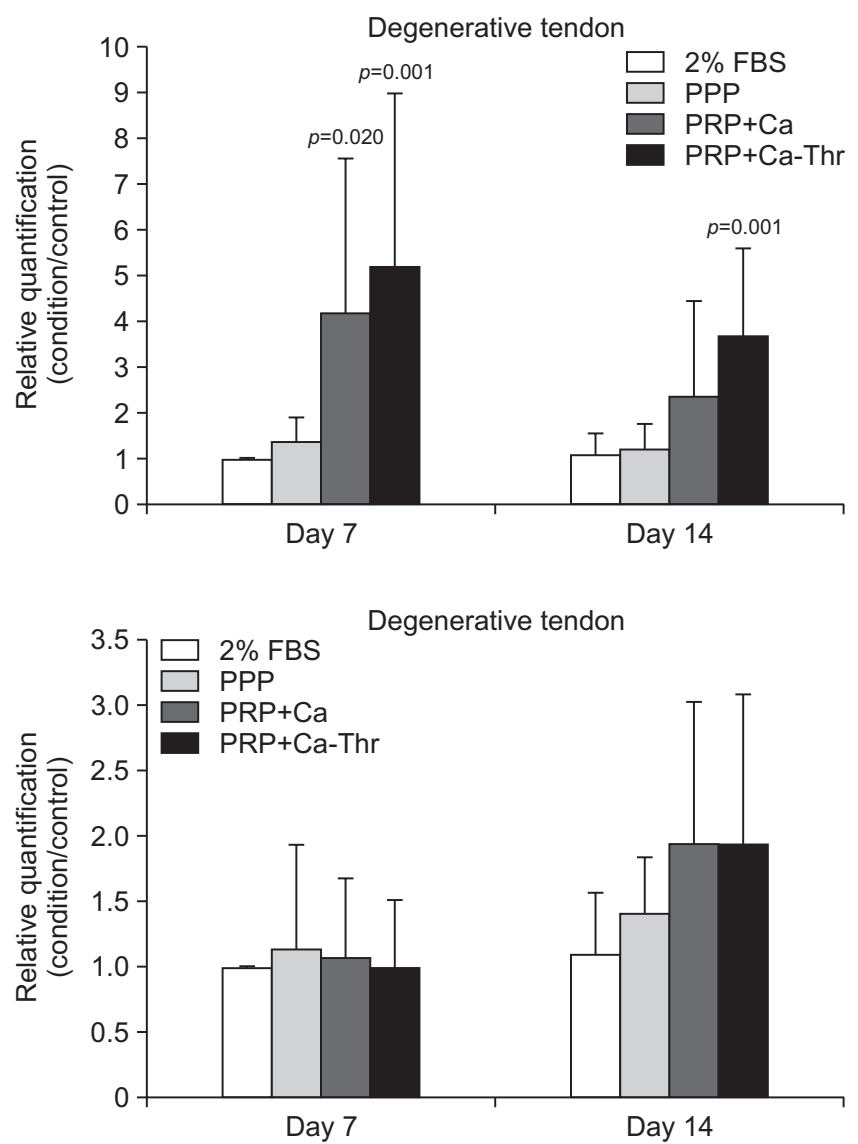

Fig. 5. Gene expression levels measured by real-time reverse transcriptase polymerase chain reaction. Relative quantifications were calculated by dividing the mRNA expression level in cells treated with platelet-rich plasma (PRP) by that in control cells. (A) Decorin. (B) Tenascin-C. (C) Scleraxis. FBS: fetal bovine serum, PPP: platelet-poor plasma, PRP-Ca: PRP activated with calcium, PRP-Ca-Thr: PRP activated with calcium and thrombin. 
as compared to control. At day 14, PRP-Ca and PRP-Ca-Thr upregulated the gene expression of type III collagen by 2.82and 3.33-fold, respectively (All $p<0.001$ ). In the normal tendon, although PRP-Ca and PRP-Ca-Thr increased the gene expression of type III collagen as compared to control, no statistically significant difference was observed due to the large variation between samples. The gene expression of type III collagen was upregulated 6.2- and 6.7-fold, respectively, in the normal tendon (Fig. 3B). No significant difference was observed in the ratio of type III/I collagen between the groups (Fig. 2C). PRP-Ca and PRP-Ca-Thr also did not significantly elevate the ratio of type III/ I collagen expression at days 7 and 14 compared to the control. In normal tendon, PRP-Ca and PRP-Ca-Thr increased the fold change values of Type III/I collagen ratio at day 14 by 3.4- and 2.82-fold, respectively. In the degenerative tendon, PRP-Ca and PRP-Ca-Thr showed minimal fold change at day 14 , being 0.71 and 0.64-fold, respectively (Fig. 3C).

There were no significant differences in the gene expression levels of decorin, tenascin-C, and scleraxis between tenocytes harvested from normal and degenerative tendon at days 7 and 14 (Fig. 4). PRP-Ca-Thr significantly induced the gene expressions of decorin and tenascin-C only in tenocytes from degenerative tendon at day 14 (All $p=0.001$, respectively), but levels remained unchanged in the normal group (Fig. 5).

\section{Effect of Platelet-rich Plasma on Total Collagen and Glycosaminoglycan Synthesis between Normal and Degenerated Torn Tendon}

For Following treatment with PRP-Ca and PRP-Ca-Thr significant differences in the total collagen production were observed between normal and degenerative tendon at days $7(p=0.026, p=0.024$, respectively) and days $14(p=0.015$, $p=0.014$, respectively) (Fig. 6A). Comparing the GAG synthesis with control, PPP, PRP-Ca and PRP-Ca-Thr, significant differences were observed between tenocytes from normal and degenerative tendon at days 7 and 14 (all $p<0.001$, except $p=0.030$, $p=0.007$ for PRP-Ca, PRP-Ca-Thr at day 7) (Fig. 6B). At day 7, the total collagen synthesis significantly increased with PPP and
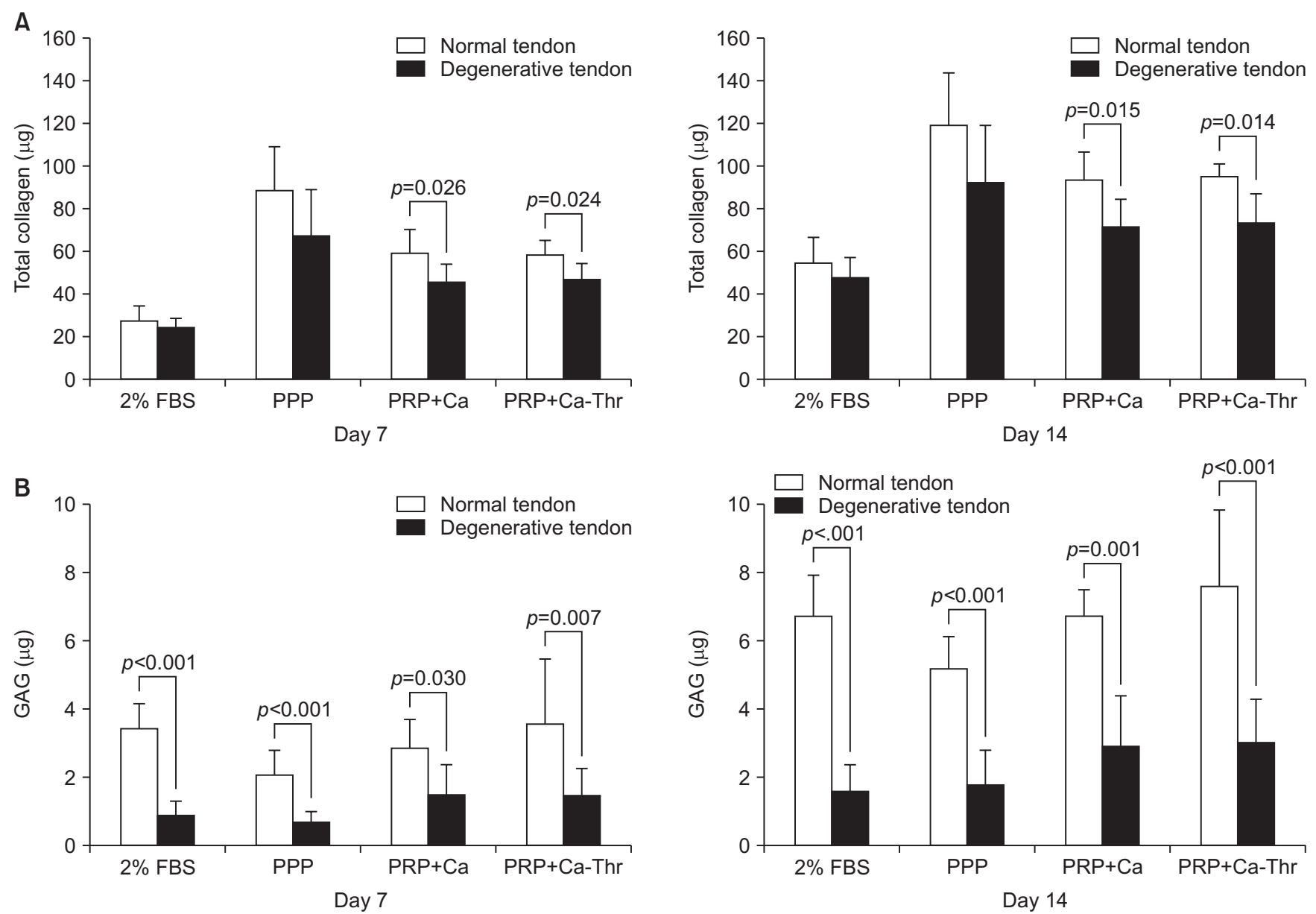

Fig. 6. Relationship between normal group and degenerative group in matrix synthesis. (A) Total collagen synthesis. (B) Glycosaminoglycan (GAG) synthesis. FBS: fetal bovine serum, PPP: platelet-poor plasma, PRP-Ca: platelet-rich plasma activated with calcium, PRP-Ca-Thr: platelet-rich plasma activated with calcium and thrombin. 
PRP-Ca in the normal group ( $p=0.001, p=0.049$, respectively) and PPP, PRP-Ca, and PRP-Ca-Thr in the degenerative group (all $p<0.001$ ). At day 14 , the total collagen synthesis significantly increased with PPP and PRP-Ca-Thr in the normal group $(p=0.003, p=0.042$, respectively) and with PPP, PRP-Ca, and PRP-Ca-Thr in the degenerative group $(p<0.001, p=0.005$, $p=0.002$, respectively) (Fig. 7A). At day 14 , the GAG synthesis in the normal group increased by 1.0- and 1.12- fold with PRPCa and PRP-Ca-Thr, respectively, and by 1.8- and 1.86 -fold with PRP-Ca and PRP-Ca-Thr, respectively, in the degenerative group, when compared with the control. The degenerative group were significantly increased with PRP-Ca and PRP-Ca-Thr at day 14 ( $p=0.032, p=0.020$, respectively), but there was no statistical difference observed in the normal group (Fig. 7B).

\section{Discussion}

The results of this study determined the following important outcomes: (1) PRP stimulated proliferation \& matrix synthesis in both tenocytes from normal human rotator cuff tendon and rotator cuff tendon with degenerative tear. (2) PRP stimulated the proliferation and gene expression of decorin, tenascin-c and GAG synthesis in tenocytes from rotator cuff tendon, being greater in degenerative tear than in normal tenocytes. (3) Foldchange of type III/I collagen ratio was lower and fold-change of GAG synthesis was higher in tenocytes from rotator cuff tendon with degenerative tear than in tenocytes from normal tendon.

Several studies have shown the difference in cell proliferation between normal tendon and ruptured tendon. Matthews et al. ${ }^{3)}$ reported that cell proliferation was increased in full-thickness rotator cuff tear of small- and medium- size than normal tendon. Lundgreen et al. ${ }^{19)}$ also reported that in the degenerative tendon, the density of tenocytes increased and the proliferation rate is higher than in normal tissue. Besides the difference between normal and degenerative tendon, Anitua et al. ${ }^{20)}$ reported that $20 \%$ PRP releasate ( $\mathrm{vol} / \mathrm{vol})$ increased the cell proliferation and production of vascular endothelial growth factor and hepatocyte growth factor in human healthy hamstring tendon. Our previous study showed that PRP activated calcium with or without thrombin significantly stimulated the proliferation of tenocytes
A

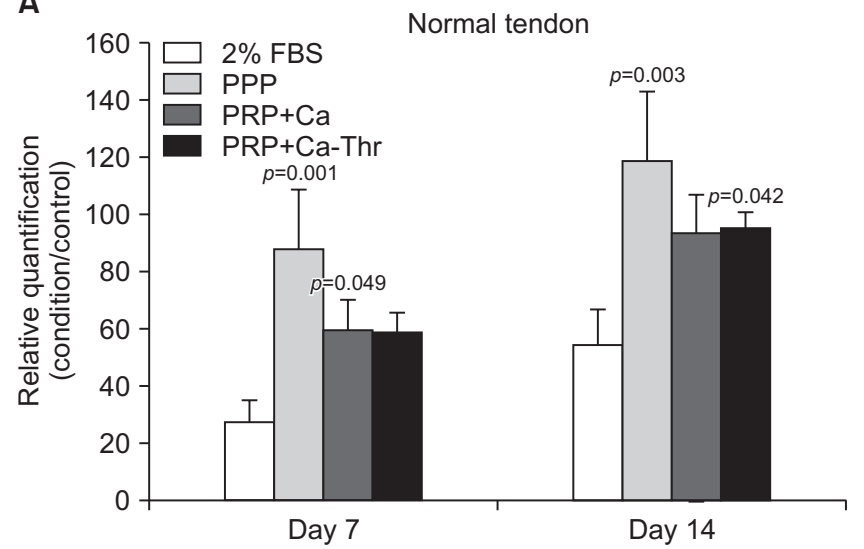

B

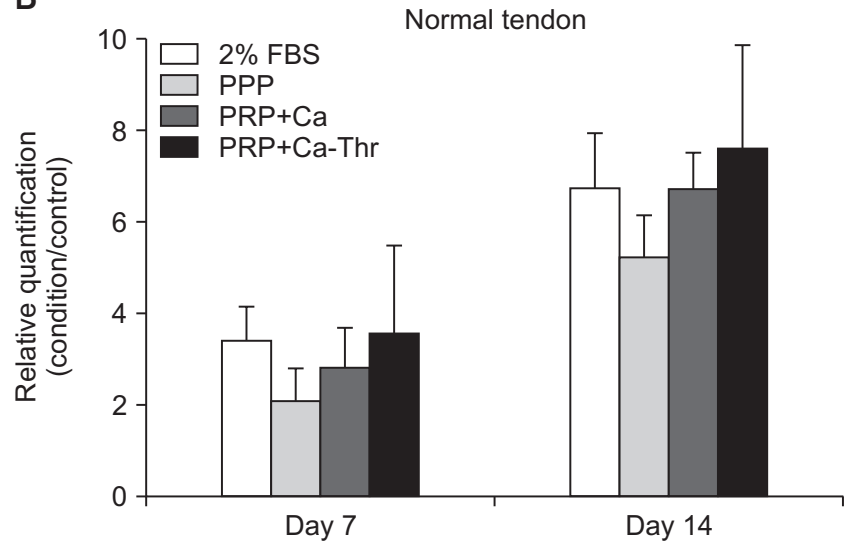

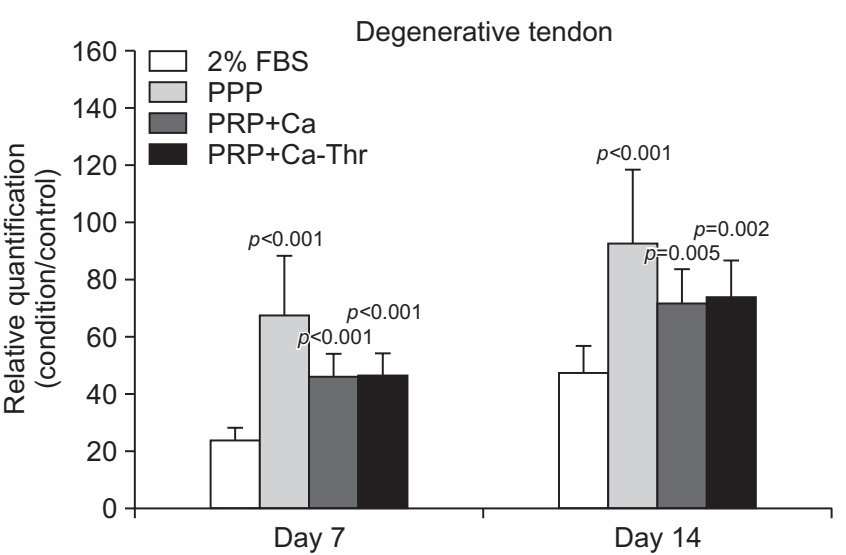

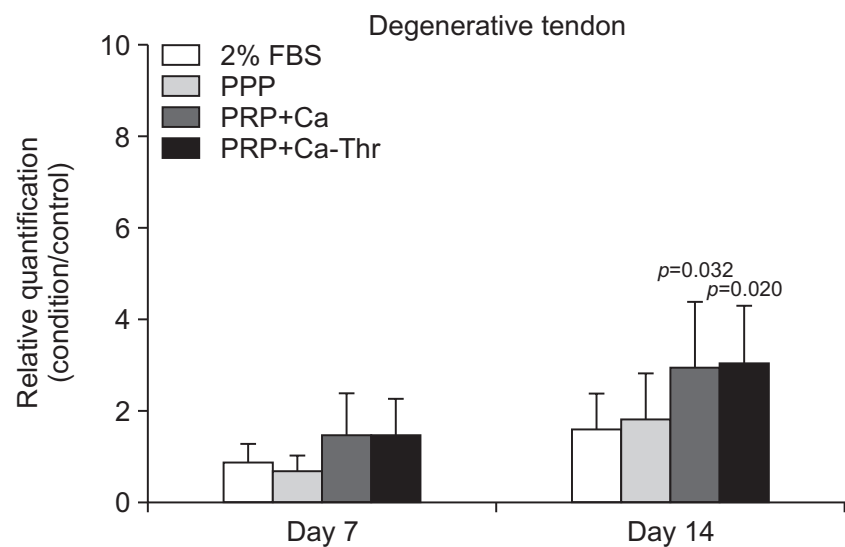

Fig. 7. (A) Total collagen synthesis measured using the Sircol assay (Biocolor, Newtownabbey, UK). (B) Glycosaminoglycan synthesis measured using the Blyscan assay (Biocolor).

FBS: fetal bovine serum, PPP: platelet-poor plasma, PRP-Ca: platelet-rich plasma activated with calcium, PRP-Ca-Thr: platelet-rich plasma activated with calcium and thrombin. 
from human rotator cuff tendons with degenerative tears in a dose-dependent manner." The results of this study show the same pattern in normal tendon. There were no significant differences between normal tendon and degenerated torn tendon on the day 7 , but on day 14 the cell proliferation was more than that seen in degenerated torn tendon at most concentrations. Yu et al. ${ }^{21)}$ reported that PRP contains high concentrations of transforming growth factor- $\beta 1$ and platelet-derived growth factor that increase the tendon cell proliferation by modulating Stat3/p27(Kip1), which enhances the expression of cyclin-Cdk complexes that promote cell cycle progression. However, there has been little research on the mechanism. ${ }^{22)}$ Although it is difficult to know the precise mechanism for better proliferation in degenerative tendons, it can be thought that PRP gives more stimulation to the components that change in the degenerative tendon.

GAG was significantly different than the control and the normal and degenerative tendon. On days 7 and 14, the GAG synthesis was increased in the normal tendon compared with degenerated torn tendon. However, in the degenerated torn tendon, the fold-change was higher than that of the normal tendon, and was statistically significant as compared with the control. Previous studies have reported that the remodeling phase occurs 6 weeks after injury of the tendon, at which time the cellularity, collagen and GAG synthesis decrease. ${ }^{23)}$ Our results showed that the synthesis rate was greater than normal. Chronic rotator cuff tear was end-stage, and we believe that the GAG synthesis decreases after a longer duration, but PRP increases the rate of synthesis on day 14 .

Lo et al. ${ }^{24)}$ reported changes in proteoglycans; aggrecan increased while decorin decreased in the ruptured rotator cuff tendon when compared with normal cadaveric controls. An increase in glycoproteins such as tenascin- $\mathrm{C}$ and fibronectin have also been reported in ruptured tendons. ${ }^{25,26)}$ Karousou et al. ${ }^{27}$ reported greater gene expression of proteoglycan core protein decorin and versican in human achilles tendon rupture. Our study showed no significant differences in the gene expression levels of decorin, tenascin- $\mathrm{C}$, and scleraxis after PRP application, between tenocytes from normal and degenerated torn tendon. However, PRP significantly increased the expression levels of decorin and tenascin- $\mathrm{C}$ in degenerated torn tendon. These results indicate that PRP has some positive effect on the damaged tendon to promote accelerated repair.

PRP enhanced the gene expression of markers for collagen type I and decreased collagen type III levels, with a resultant positive collagen $\mathrm{I} / \mathrm{III}$ ratio in equine tendon explants. ${ }^{10)}$ When PRP was administered to normal tendon of children aged 13 to 15 years, de Mos et al. ${ }^{9)}$ demonstrated that PRP releasate increased the cell number and total collagen levels, but decreased the gene expressions of type I and III collagen without affecting the III/I ratio. Our previous study showed that PRP significantly increased the gene expression of type III collagen, but maintained the type III/I ratio in tenocytes from human rotator cuff tendons with degenerative tears. ${ }^{7)}$ Variations in results could arise because tendons from different sites have different structures, compositions, cell phenotypes, and metabolic characteristics, and the behavior of tenocytes are known to depend on donor age, anatomic site or origin, and status from intact, injured, or degenerated tendons. ${ }^{28-30}$ In this current study, the effect of PRP was different from that of de Mos et al.,. ${ }^{9)}$ since the gene expression of type I and III collagen in both tenocytes from normal and degenerative tendon were increased, and we showed that there were no statistically significant differences between normal and degenerative tendon. The gene expression of type III collagen was only significantly increased in degenerative tendon. However, the fold-change of type I collagen in degenerative tendon was higher than in normal tendon. Although there were no statistical significances, this increased rate can be considered to reflect a greater impact of PRP on the degenerative tendon.

One limitation of the present study is that normal tendon is not abundant. Practically, it is difficult to obtain normal tendons from age-matched patients without a rotator cuff tear. Also, normal tendons were obtained from proximal humerus fractures. These tendons could not be considered representative of normal tissue because of microscopic tears of tendon or age-related degenerative changes. Furthermore, we used the normal tendon with no shoulder related trauma or symptoms based on patient history and macroscopically normal. However, ethical reasons and the relatively small amount of tendons made it difficult to analyze the normal tendon in several assays.

Another limitation is that we harvested small pieces of tendon after removing the overlaying synovium, after which the tissue was finely minced. During the procedure, any suspicious tissue other than tendon was removed. Hence, the majority of cells used in the study would be tenocytes. Based on this study, additional studies are required simulating different clinical situations.

\section{Conclusion}

PRP promotes the proliferation of tenocytes in both normal rotator cuff tendon and degenerative torn tendon, and enhances the synthesis of the tendon matrix. PRP has a more positive effect on cell proliferation, matrix gene expression and synthesis in tenocytes from degenerative torn tendon than tenocytes from normal tendon. The results of this study might provide a useful biological strategy for promoting the regeneration of rotator cuff tears.

\section{References}

1. Urwin M, Symmons D, Allison T, et al. Estimating the burden of musculoskeletal disorders in the community: the compara- 
tive prevalence of symptoms at different anatomical sites, and the relation to social deprivation. Ann Rheum Dis. 1998; 57(11):649-55.

2. Jo CH, Shin WH, Park JW, Shin JS, Kim JE. Degree of tendon degeneration and stage of rotator cuff disease. Knee Surg Sports Traumatol Arthrosc. 2017;25(7):2100-8.

3. Matthews TJ, Hand GC, Rees JL, Athanasou NA, Carr AJ. Pathology of the torn rotator cuff tendon. Reduction in potential for repair as tear size increases. J Bone Joint Surg Br. 2006;88(4):489-95.

4. Chaudhury S, Carr AJ. Lessons we can learn from gene expression patterns in rotator cuff tears and tendinopathies. J Shoulder Elbow Surg. 2012;21(2):191-9.

5. Riley GP. Gene expression and matrix turnover in overused and damaged tendons. Scand J Med Sci Sports. 2005;15(4): 241-51.

6. Boswell SG, Schnabel LV, Mohammed HO, Sundman EA, Minas T, Fortier LA. Increasing platelet concentrations in leukocyte-reduced platelet-rich plasma decrease collagen gene synthesis in tendons. Am J Sports Med. 2014;42(1):42-9.

7. Jo CH, Kim JE, Yoon KS, Shin S. Platelet-rich plasma stimulates cell proliferation and enhances matrix gene expression and synthesis in tenocytes from human rotator cuff tendons with degenerative tears. Am J Sports Med. 2012;40(5):1035-45.

8. Bosch G, van Schie HT, de Groot MW, et al. Effects of platelet-rich plasma on the quality of repair of mechanically induced core lesions in equine superficial digital flexor tendons: a placebo-controlled experimental study. J Orthop Res. 2010;28(2):211-7.

9. de $\operatorname{Mos} M$, van der Windt $A E$, Jahr $H$, et al. Can platelet-rich plasma enhance tendon repair? A cell culture study. Am J Sports Med. 2008;36(6):1171-8.

10. McCarrel T, Fortier L. Temporal growth factor release from platelet-rich plasma, trehalose lyophilized platelets, and bone marrow aspirate and their effect on tendon and ligament gene expression. J Orthop Res. 2009;27(8):1033-42.

11. Anitua E, Andía I, Sanchez M, et al. Autologous preparations rich in growth factors promote proliferation and induce VEGF and HGF production by human tendon cells in culture. J Orthop Res. 2005;23(2):281-6.

12. Muto T, Kokubu T, Mifune $Y$, et al. Effects of platelet-rich plasma and triamcinolone acetonide on interleukin-1 $\beta$ stimulated human rotator cuff-derived cells. Bone Joint Res. 2016;5(12):602-9.

13. Wang X, Qiu Y, Triffitt J, Carr A, Xia Z, Sabokbar A. Proliferation and differentiation of human tenocytes in response to platelet rich plasma: an in vitro and in vivo study. J Orthop Res. 2012;30(6):982-90.

14. Jo CH, Lee SY, Yoon KS, Shin S. Effects of platelet-rich plasma with concomitant use of a corticosteroid on tenocytes from degenerative rotator cuff tears in interleukin $1 \beta$-induced tendinopathic conditions. Am J Sports Med. 2017;45(5):1141-50.

15. Cross JA, Cole BJ, Spatny KP, et al. Leukocyte-reduced plateletrich plasma normalizes matrix metabolism in torn human rotator cuff tendons. Am J Sports Med. 2015;43(12):2898-906.

16. Alsousou J, Thompson M, Harrison P, Willett K, Franklin S. Effect of platelet-rich plasma on healing tissues in acute ruptured Achilles tendon: a human immunohistochemistry study. Lancet. 2015;385 Suppl 1:S19.

17. Jo $\mathrm{CH}$, Kim JE, Yoon KS, et al. Does platelet-rich plasma accelerate recovery after rotator cuff repair? A prospective cohort study. Am J Sports Med. 2011;39(10):2082-90.

18. Jo $\mathrm{CH}$, Kim OS, Park EY, et al. Fetal mesenchymal stem cells derived from human umbilical cord sustain primitive characteristics during extensive expansion. Cell Tissue Res. 2008;334(3):423-33.

19. Lundgreen K, Lian OB, Engebretsen L, Scott A. Tenocyte apoptosis in the torn rotator cuff: a primary or secondary pathological event? Br J Sports Med. 2011;45(13):1035-9.

20. Anitua E, Sánchez M, Orive G, Andía I. The potential impact of the preparation rich in growth factors (PRGF) in different medical fields. Biomaterials. 2007;28(31):4551-60.

21. Yu TY, Pang JH, Wu KP, Lin LP, Tseng WC, Tsai WC. Plateletrich plasma increases proliferation of tendon cells by modulating Stat3 and p27 to up-regulate expression of cyclins and cyclin-dependent kinases. Cell Prolif. 2015;48(4):413-20.

22. Masoudi E, Ribas J, Kaushik G, Leijten J, Khademhosseini A. Platelet-rich blood derivatives for stem cell-based tissue engineering and regeneration. Curr Stem Cell Rep. 2016;2(1):3342.

23. Sharma $P$, Maffulli N. Tendon injury and tendinopathy: healing and repair. J Bone Joint Surg Am. 2005;87(1):187-202.

24. Lo IK, Boorman R, Marchuk L, Hollinshead R, Hart DA, Frank CB. Matrix molecule mRNA levels in the bursa and rotator cuff of patients with full-thickness rotator cuff tears. Arthroscopy. 2005;21(6):645-51.

25. Riley GP, Harrall RL, Cawston TE, Hazleman BL, Mackie EJ. Tenascin-C and human tendon degeneration. Am J Pathol. 1996;149(3):933-43.

26. Tillander B, Franzén L, Norlin R. Fibronectin, MMP-1 and histologic changes in rotator cuff disease. J Orthop Res. 2002;20(6):1358-64.

27. Karousou E, Ronga M, Vigetti D, Passi A, Maffulli N. Collagens, proteoglycans, MMP-2, MMP-9 and TIMPs in human achilles tendon rupture. Clin Orthop Relat Res. 2008;466(7):1577-82.

28. Franchi $M$, Trirè A, Quaranta $M$, Orsini E, Ottani V. Collagen structure of tendon relates to function. ScientificWorldjournal. 2007;7:404-20.

29. Goodman SA, May SA, Heinegård D, Smith RK. Tenocyte response to cyclical strain and transforming growth factor 
Clinics in Shoulder and Elbow

Vol. 21, No. 1, March, 2018

beta is dependent upon age and site of origin. Biorheology. 2004;41(5):613-28.

30. Young NJ, Becker DL, Fleck RA, Goodship AE, Patterson-Kane
JC. Maturational alterations in gap junction expression and associated collagen synthesis in response to tendon function. Matrix Biol. 2009;28(6):311-23. 